\title{
Archéopages
}

Archéopages

Archéologie et société

34 | 07/2012

Campagnes

\section{Représenter des mobiliers complexes grâce à la 3D}

\section{Pascal Raymond}

\section{CpenEdition}

Journals

Édition électronique

URL : https://journals.openedition.org/archeopages/421

DOI : $10.4000 /$ archeopages.421

ISSN : 2269-9872

\section{Éditeur}

INRAP - Institut national de recherches archéologiques préventives

\section{Édition imprimée}

Date de publication : 1 février 2012

Pagination : 93-97

ISSN : 1622-8545

\section{Référence électronique}

Pascal Raymond, «Représenter des mobiliers complexes grâce à la 3D », Archéopages [En ligne], 34 |

07/2012, mis en ligne le 01 juillet 2012, consulté le 25 janvier 2022. URL : http://

journals.openedition.org/archeopages/421 ; DOI : https://doi.org/10.4000/archeopages.421 
diagnostic, se complétaient, il a été possible de calculer un modèle $3 \mathrm{D}$ de l'intérieur de cette enceinte. La compilation des deux modèles permettra de proposer une vision globale et inédite de cette portion d'enceinte, malgré le décalage de ces interventions dans le temps et dans l'espace. Enfin, ce type de relevé a un intérêt pour la sécurité et pour l'organisation du travail. Il peut être effectué à distance de zones dangereuses à relever manuellement et il limite le temps de présence dans le sondage : de fait, le temps de prise de clichés est plus court que celui d'un relevé traditionnel dessiné, ce qui diminue en conséquence le temps d'attente des terrassiers. Ainsi, ces deux activités alternent avec une plus grande efficacité.

L'église Saint-Pierre-Saint-Paul de Gonesse La fouille de cette église gothique du Val-d'Oise a été effectuée en deux phases ${ }^{1}$. La première eut lieu dans le cœur et le déambulatoire de l'abside. Durant l'été 2012, l'intervention s'étendra à la nef. Un premier modèle numérique a été réalisé à partir de cinq campagnes de topographies et de relevés par photogrammétrie. Ce document restitue un instant « $\mathrm{T}$ » de la fouille, où le principal niveau de sépultures a été dégagé [ill. 2]. Cette opération archéologique se limitant au sous-sol, aucun relevé de bâti n'était prévu. Mais la reconstruction tridimensionnelle a permis un relevé architectural intégrant les voûtes et les différents éléments qui entourent les zones fouillées. Elle a mis en évidence le tracé régulateur sur le chœur de l'église et a permis d'établir la position de son centre théorique 2 . Cette information, essentielle dans ce contexte, facilitera la mise en perspective des fondations découvertes avec l'édifice. Ces relevés peuvent donc être replacés dans le volume entier du chœur. Les moulures des voûtes, des bases de colonnes ou des chapiteaux sont visibles et exploitables pour toutes sortes d'observations. La première phase consistait surtout à fouiller un ensemble de tombes accumulées durant près de dix siècles ; ce type de fouille s'effectue essentiellement à plat, avec prises de données topographiques et altimétriques nombreuses pour conserver un maximum d'informations sur la position des fosses sépulcrales et des squelettes. Aucune berme ne peut aisément être conservée et les relevés en coupe sont rares. La modélisation permet, là encore, de réaliser a posteriori ces profils de façon rapide, précise et pertinente, qu'il s'agisse de relevés entre sépultures ou intégrant le volume général du bâtiment [ill. 3]. De la même façon qu'une photographie générale, ces MNS conservent toutes les informations sur le relief et les volumes des vestiges. Ils constituent ainsi une base de données pour analyser les relations altimétriques des structures et effectuer des mesures dans les trois dimensions. Plus simplement, ils laissent la possibilité de naviguer dans le modèle pour visualiser l'environnement lors de la fouille ou des interventions ultérieures.
Représenter des mobiliers complexes grâce à la $3 \mathrm{D}$

Pascal Raymond, Inrap

Le mobilier lithique de grandes dimensions, lourd et encombrant, est particulièrement complexe à relever. Il doit néanmoins être représenté dans son contexte, notamment par rapport au plan horizontal [ill. 1], mais également en vue géométrale selon sa morphologie. De toutes les méthodes disponibles, la modélisation $3 \mathrm{D}$ paraît la plus appropriée et simplifie la réalisation de cette documentation. Par ailleurs, le souci d'accessibilité des vestiges ne s'explique pas seulement par le caractère destructeur de notre activité, mais parfois par des raisons économiques, légales ou physiques, notamment pour les mégalithes. L'un des intérêts de cette modélisation est également le retour possible à une information initiale d'une grande richesse.

\section{La modélisation de mégalithes gravés} Trois des blocs de grès gravés découverts à l'occasion d'un diagnostic à Citry (Seine-et-Marne) (Adrot, 2012), ont ainsi été modélisés in situ, puisqu'ils ne pouvaient pas être déplacés (surface de $4 \mathrm{~m}^{2}$ et poids de près de 3,5 tonnes pour le plus volumineux). Lors d'un diagnostic, la collecte de l'information doit se faire rapidement. Par ailleurs, l'étude se fondant sur l'échantillonnage, elle doit concilier le besoin d'identification avec la nécessité de ne pas endommager un éventuel site. Enfin, l'étude de ce type de vestiges nécessite des moyens (transport adapté, temps nécessaire au prélèvement et à l'analyse) qui n'ont pu être mis en œuvre dans ce cadre. La nécessité de reporter certaines observations imposait d'autant plus un enregistrement rigoureux de leur géométrie que le projet d'aménagement (carrière) pouvait être annulé ou modifié, ce qui aurait rendus inaccessibles ces blocs durant une période indéterminée. La modélisation $3 \mathrm{D}$ des blocs a donc été utilisée pour l'enregistrement de leur proportion avec un niveau de précision permettant la restitution des principales gravures. Avec une moyenne de 40 photographies par modèle $3 \mathrm{D}$, nous avons produit deux $\mathrm{MNS}^{\mathbf{3}}$ pris sur le terrain et deux autres après nettoyage des parties du bloc 34-15, cassé lors du diagnostic [ill. 2]. Associées à ces relevés, des portions gravées de $0,5 \mathrm{~m}^{2}$ ont fourni une documentation supplémentaire avec une résolution inférieure au millimètre sur ce qui correspond, pour chaque modèle, à un nombre de polygones compris entre 1 et 1,5 million. À partir de ces documents, il est possible de faire ressortir les gravures pour fournir des illustrations préalables à leur étude. La surface de l'un des blocs est représentée ici avec les couleurs enregistrées grâces aux photographies, mais également avec une teinte neutre en faisant varier la source d'éclairage [ill. 3.a, b, c]. Un autre mode de représentation révèle également les 

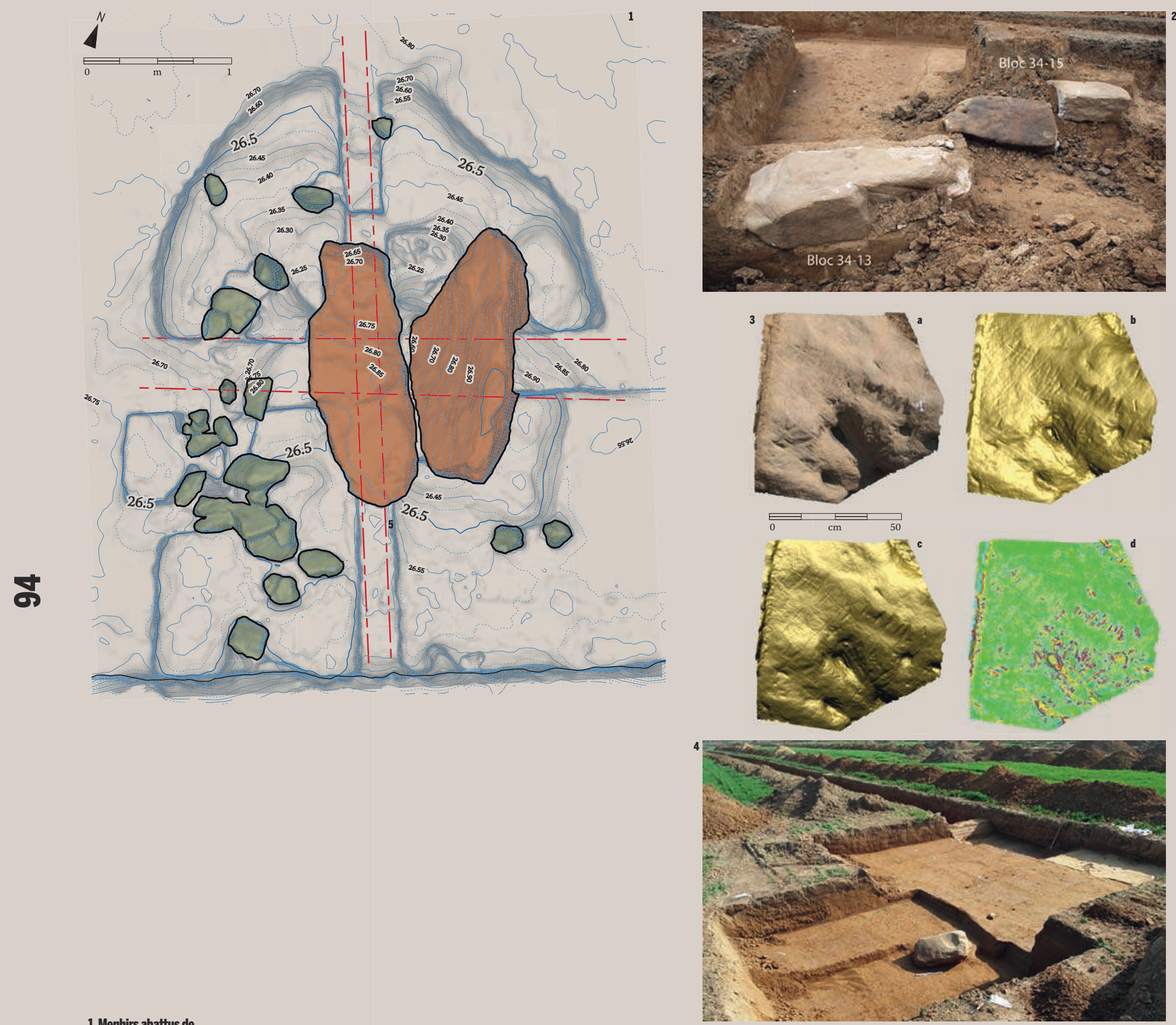

1. Menhirs abattus de

Champagnes-sur-Oise. Plan topographique des blocs en cours de foullle. Illustration obtenue par photogrammétrie et géo-référencement par

un levé topographique.

2. Vue des blocs gravés lors de leur découverte en contexte de diagnostic.

3. Détail des gravures du bloc № 34-15 modélisées d'après les photographies prises sur le terrain.

a. en couleurs vraies:

b. et c. en lumière rasante;

d.affichage des rayons

de courbure.

4. Vue du bloc de grès aménagé

en meule découvert sur

l'opération de Ballancourt-

sur-Essonne, dégagé lors

du diagnostic.

5. Représentation géométrale

et coupes du bloc de grès de Ballancourt-sur-Essonne.

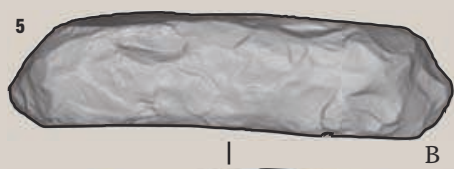

coupe longitudinale face $\mathrm{C}$
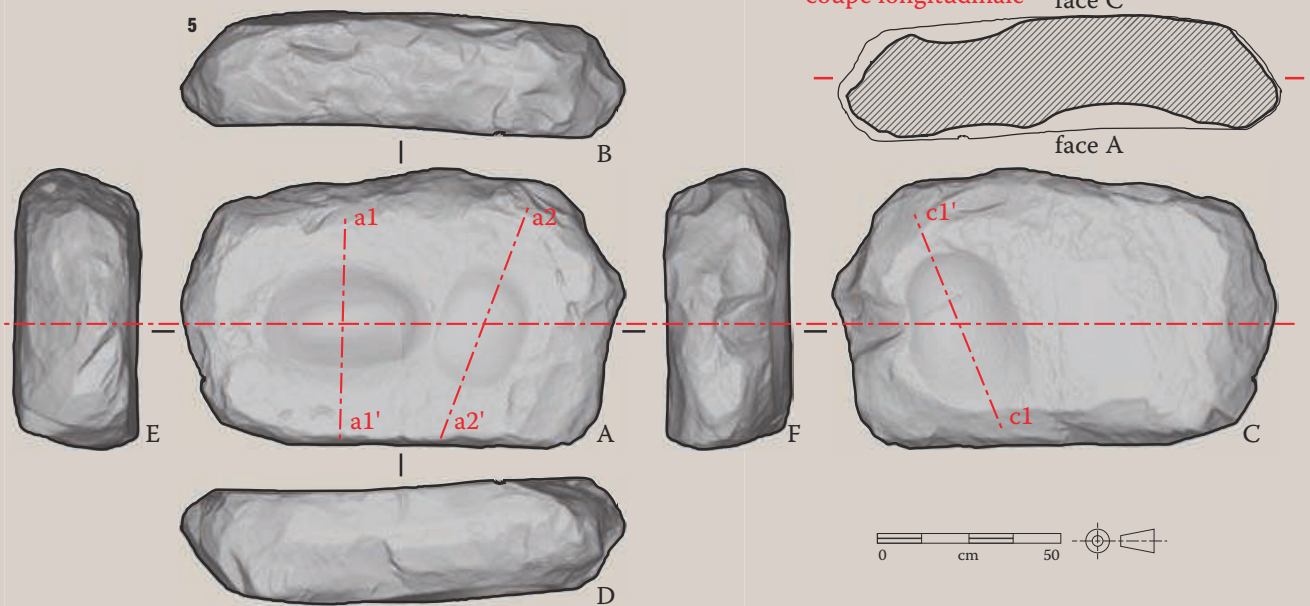

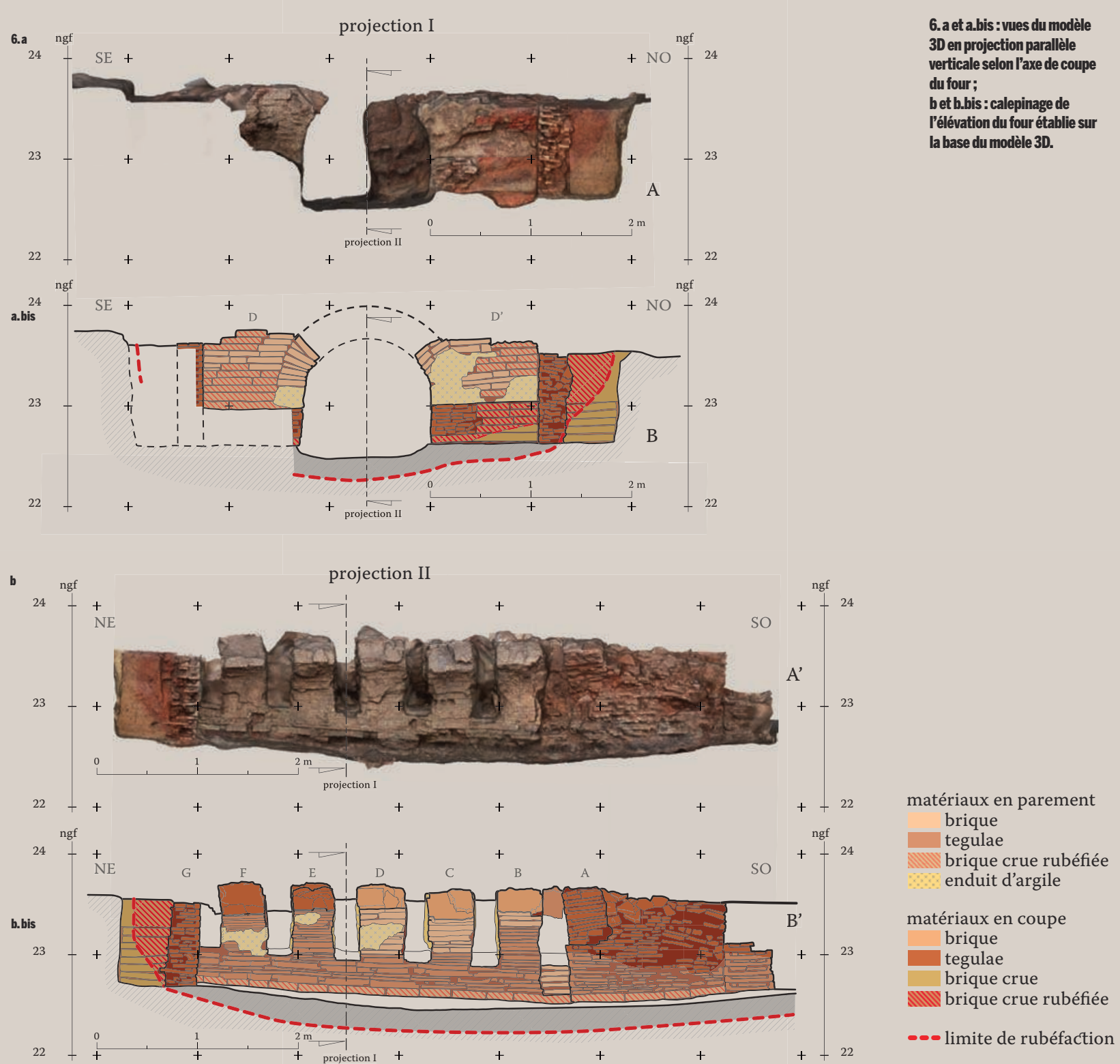

1. Meule étudiée par Cécile Monchablon (Inrap) découverte en 2012 sur le diagnostic de Ballancourt-sur-Essonne (responsable d'opération Emmnuelle Saron, Inrap). anomalies du relief par l'analyse de la surface qui fait ressortir les rayons de courbures [ill. 3.d]. Dans le meilleur des cas, ces gravures sont dessinées manuellement sur un support transparent plaqué sur le bloc. L'irrégularité du support produit une déformation du relevé qui, une fois remis à plat, génère inévitablement des anamorphoses. La méthode proposée est une autre approche basée sur la figuration des principales gravures par leur restitution numérique dans un plan de projection paramétré. Ces documents fournissent une base topographique à partir de laquelle une analyse pourra être réalisée. En dissociant le relevé de l'étude, on peut mieux distinguer ce qui est géométriquement juste de ce qui est authentique d'un point de vue anthropique.

Les meules aménagées sur un bloc de grès du Néolithique final découvertes à Ballancourt-surEssonne ${ }^{\mathbf{1}}$ (Essonne) (Saron, 2012) illustrent un autre exemple de mobilier difficile à dessiner [ill. 4].
La diffusion de l'information passe par sa codification. La figuration des faits répond donc à des conventions qui s'adaptent à une nécessité d'analyse comme pour le dessin lithique, la céramique ou les différents types de mobilier. Ce qui caractérise le dessin archéologique de façon générale, c'est l'application des normes graphiques de la géométrie descriptive et principalement la forme géométrale. Ainsi, la projection parallèle demeure la mieux adaptée à la prise de cotes sur le dessin, ce qui facilite les comparaisons. Cependant, cette mise à plat du mobilier demeure un exercice difficile lorsque celui-ci est volumineux.

Bien que la forme du bloc de Ballancourt s'inscrive dans un parallélépipède, la projection de chacune de ses six faces selon des plans perpendiculaires les uns aux autres est un travail ardu. Deux faces d'utilisation sont aménagées avec des meules et des surfaces polies. Le bloc présente aussi une série d'éclats d'épannelage et ses dimensions, $125 \times 80 \times 35 \mathrm{~cm}$, pour un poids de $475 \mathrm{~kg}$, interdisent 
1. Illustration du protocole

des prises de vues.

2. Modélisation d'un contenant

hypothétique sur une

acquisition tridimensionnelle d'incinération.

3. Coupe et mesure réalisées

avec Adobe Acrobat sur une

incinération modélisée en 3D.
ஜ

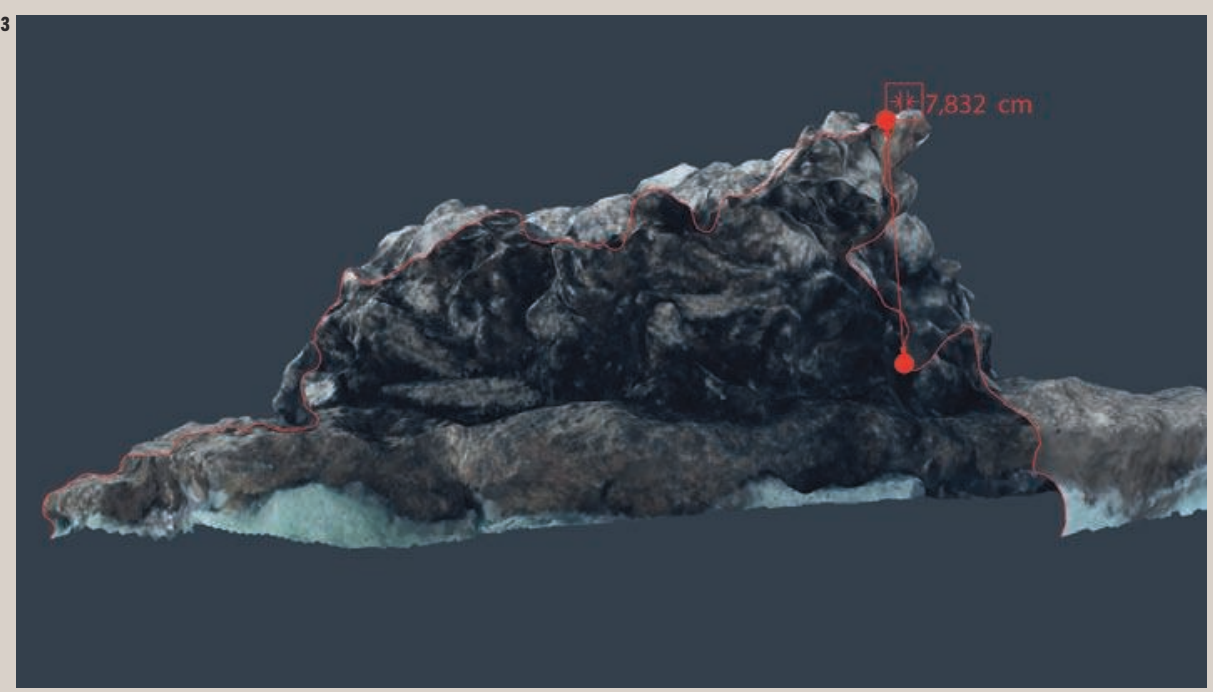


sa manipulation sans moyen de levage. Réalisées en deux phases, 133 photos ont produit deux MNS regroupés en un modèle de 2,34 millions de faces avec une résolution de $1,4 \mathrm{~mm}$. Ce document est assez précis pour restituer les éclats, les cupules de broyage et jusqu'aux détails des principales marques de bouchardage. Ces caractéristiques morphologiques et la qualité des traces d'utilisation ont ainsi justifié la modélisation du bloc afin d'en extraire des vues orthogonales [ill. 5]. La 3D a également facilité la création de coupes et profils selon des plans paramétrés ou libres pour fournir des informations complémentaires. Les illustrations obtenues constituent une base graphique géométriquement fiable sur laquelle les observations et les résultats d'analyse peuvent être reportés.

Le modèle ${ }_{3} D$ comme support à l'enregistrement Deux étapes de travail doivent être distinguées : d'une part, l'enregistrement des géométries et, d'autre part, l'interprétation des vestiges. La prise de cotes par des moyens traditionnels accapare une part importante du temps qui pourrait être affecté à l'étude. En dissociant ces deux tâches et en éliminant au maximum la part d'interprétation lors des prises de mesure, on crée un cadre plus juste pour l'analyse. Grâce à sa souplesse pour réaliser des vues géométrales, la modélisation permet d'extraire des images en projection orthogonale qui servent ensuite de support à un relevé interprété.

Nous avons procédé de cette façon pour un four de tuilier ${ }^{1}$ découvert à Mours (Val-d'Oise) (Mondoloni, à paraître). Les coupes longitudinales et transversales de l'alandier, de la chambre de chauffe et de la maçonnerie ont été modélisées en un MNS de 1,47 million de faces. Ce document, très riche et géométriquement juste, a été projeté selon les axes de coupes et imprimés au $1 / 20^{\mathrm{e}}$ [ill. 6 .a et a.bis]. Ces représentations ont permis l'enregistrement des gestes du constructeur sur un calque en retombe. Ainsi, nous avons produit un plan de calepinage qui décrit les différents matériaux [ill. 6.b et b.bis], les phases de construction et leurs réparations. La phase de description sur le terrain a été plus courte et facilitée par ces supports imprimés grâce auxquels il nétait plus utile de prendre des mesures pour faire figurer les différents éléments.

\section{La modélisation d'incinérations}

Nicolas Saulière, Inrap

Les premiers essais de la photogrammétrie en archéologie réalisés par les équipes Inrap d'Îlede-France ont porté sur des structures complexes (four de tuilier, sépulture...), mais aussi sur des objets de grandes dimensions (menhirs). Cette technique de modélisation tridimensionnelle a été réalisée sur des objets de moindre échelle lors de l'étude de la nécropole de la « Plaine du moulin à Vent » à Cesson (Seine-et-Marne). Sur ce site furent mises au jour 170 sépultures secondaires à crémation, datées de l'âge du Bronze moyen et final. Notre collègue anthropologue Anne-Gaëlle De Kepper a suggéré d'en modéliser un échantillon. En effet, la majorité des dépôts cinéraires étant constituée d'amas osseux dont le contenant en matériau périssable n'a pas été conservé, les modèles $3 \mathrm{D}$ devaient servir à en restituer la morphologie initiale. Les résultats obtenus permettront de constituer une base de données pour ce type de dépôt. L'étude étant en cours, seule une ébauche des résultats est présentée ici. Sont abordées la méthodologie adoptée pour la modélisation, les contraintes dues à ce type de mobilier, et les études envisagées.

Deux types de modèles ont été réalisés en fonction de l'état de conservation des dépôts cinéraires. Si les ossements sont très fragmentés et non identifiables sur place, le modèle sert à une étude morphologique de l'amas osseux, afin de déterminer la forme du contenant. Dans ce cas, le nombre de photographies a été limité afin d'obtenir des modèles relativement légers et d'optimiser le temps de calcul. Mais si les ossements sont bien conservés et identifiables, il est aussi possible de voir leur répartition au sein de l'amas selon les différentes régions anatomiques du corps humain (crâne, tronc, membres). Le nombre de clichés augmente alors selon l'importance accordée aux détails. Après prélèvement et étude en laboratoire de l'amas osseux, l'incinération est installée de façon à effectuer une couverture photographique complète de la structure. Le protocole consiste en quatre prises de vue à la verticale du sujet, légèrement décalées les unes des autres afin de s'adapter à la contrainte de la forme cylindrique des incinérations et à la nécessité de réaliser des prises de vue se recouvrant par moitié ; puis furent réalisés plusieurs clichés en spirale autour de l'incinération [ill. 1]. Entre 40 et 90 photographies ont ainsi été réalisées pour chaque incinération en fonction de la résolution visée. Plusieurs contraintes optiques sont à noter. Tout d'abord, plus les objets sont petits, plus il est difficile d'avoir une bonne profondeur de champ. Or les photographies comportant des plans flous génèrent du «bruit » (points parasites n'ayant aucune position cohérente). Par ailleurs, il est nécessaire de corriger les effets des inévitables situations de contre-jour, puisque, en tournant à $360^{\circ}$ autour des incinérations, il arrive d'être face à la source de lumière. Il convient alors de légèrement surexposer les photographies pour éviter d'avoir un sujet trop sombre. Malgré ces précautions, il a fallu opérer un important travail d'épuration sur les nuages de points avant de générer, à partir du logiciel Meshlab, un maillage de polygones. La morphologie des incinérations a été étudiée à l'aide du logiciel Blender qui permet de créer des formes géométriques et de les comparer au modèle issu de Meshlab. On peut, de la sorte,
1. Fouille « Le derrière des Moulins» : Alexandra Mondoloni, Inrap, 2011/2012; étud du four : Aurélia Aligri, Inrap. 